\title{
Predicting acute hypotensive episodes from ambulatory blood pressure telemetry
}

\author{
Kun Jin*,† And Norman StockBridge*
}

The biological data collected from intensive care units contain signal and noise. To extract information that will be useful for predicting or discriminating the cases likely to develop an acute hypotensive episode (AHE), we begin by applying a spline-based smoothing method to the observed mean arterial pressure (MAP) curves. The coefficients of the fitted spline model form a discretization matrix of the continuous MAP curves. A rank-based discriminant analysis and a cross-validation method are developed to find the best prediction subset in the training set. The selected best subsets are used to predict AHE in the test sets. This work is from participation of PhysioNet/Computers in Cardiology Challenge 2009: Predicting Acute Hypotensive Episodes.

AMS 2000 SUbJeCt Classifications: Primary 62P10, 6207; secondary 65D07, 65D10.

KeYWORDS AND PHRASES: B-spline smoothing, Rank analysis, Cross validation, Acute hypertensive episode, Ambulatory blood pressure telemetry.

\section{INTRODUCTION}

An acute hypotensive episode (AHE), which is defined as any period of 30 or more minutes during which at least 90 percent of mean arterial blood pressure measurements are less than $60 \mathrm{mmHg}$, requires urgent intervention to prevent ischemic complications at the intensive care unit (ICU). False positive alarms, however, waste care resources and drive up costs of medical care. The goal of Computers in Cardiology Challenge 2009 (Moody and Lehman, 2009) was to predict AHEs occurring within a 60 -minute window following a day of observation. The Challenge provided three datasets: a training set and test sets $\mathrm{A}$ and $\mathrm{B}$. The training set has data collected from 60 patients, of whom 30 patients developed an AHE during the 60-minute forecast window. Test set A has data from 10 patients, and one is supposed to predict the 5 patients who had AHEs from this set. Test set B has data from 40 patents, and one is supposed to predict the 10 to 16 patients who had AHEs from this set. The detailed description of the datasets can be found at http:// www.physionet.org/challenge/2009/.

* The views expressed in this manuscript are those of the authors and not necessarily those of the Food and Drug Administration.

${ }^{\dagger}$ Corresponding author.
All datasets include continuous telemetric data for heart rate, systolic and diastolic pressure, and mean arterial blood pressure (MAP), and other clinical information on vital signs, concomitant medications, etc. In this article, only the mean blood pressure data were used to predict AHE. Figure 1 plots four patients' data from the Training Set. The top two plots are mean blood pressure curves for patients who developed an AHE in the forecast window with the starting point $T_{0}$. The bottom two are patients who had no AHE. The data were collected from intensive care units at hospitals; there are significant segments of missing data, for example, in the bottom two curves. In the Training Set, all but one patient has at least 656 minutes of continuous data immediately before the starting point $T_{0}$. The missing data are interruptions in telemetry for unknown reasons. In this paper, no attempt was made to impute missing data. Our aim was to predict episodes with existing data only.

In this paper, we propose a two-stage approach to this challenge. The first step is to approximate the mean arterial blood pressure curve by cubic B-Splines. The resulting coefficients corresponding to the bases are considered to be a discretization of the continuous MAP. The second step is to find the "best subset" of the discretization from the training set that discriminates patients having an AHE. This "best subset" is then used to predict who has an AHE from the two test sets.

\section{CUBIC B-SPLINES AND DISCRETIZATION}

Cubic B-Splines (de Boor, 2001) with equally spaced knots are used to approximate MAP curves. The selection of B-Splines is more or less arbitrary. It is anticipated similar approximation methods, such as wavelets, could do as well as B-Splines. Figure 2 plots a cubic B-spline basis with three internal knots. With appropriate empirical parameter selection, B-Splines approximate target curves well and can capture local features of the target curve (Jin, 1992). Technically, the t-axis of original MAP curves is inverted so that $t=0$ at $T_{0}$, the starting point of the prediction window.

Let $B_{k}(t), k=1, \ldots, K$ be bases with $K$ knots. Denote $A B P M_{i}\left(t_{j}\right), j=1, \ldots, n_{i}$ to be $i^{t h}$ MAP curve. $\sum_{i=1}^{K} \widehat{\alpha_{i k}} B_{k}\left(t_{j}\right)$ is used to approximate the MAP curve and 
$\mathrm{H}$

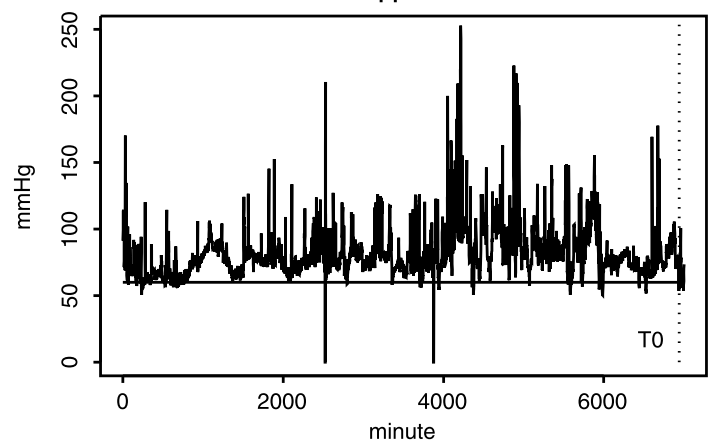

C

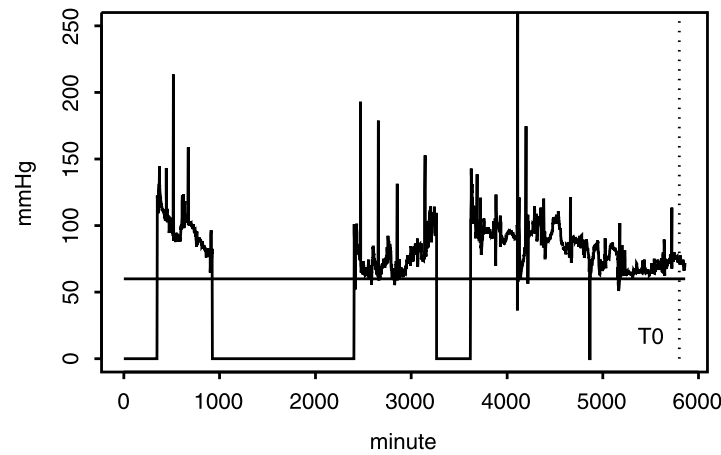

$\mathrm{H}$

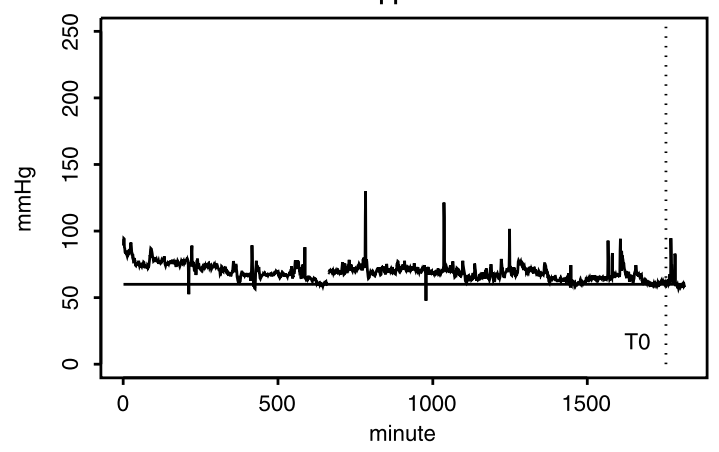

C

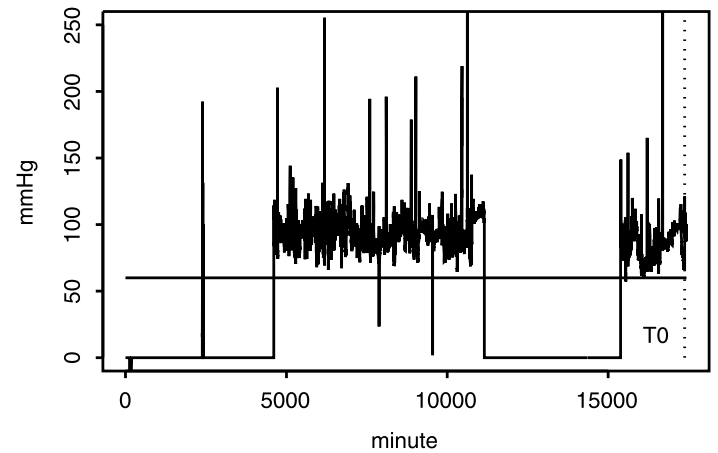

Figure 1. Plot of mean blood pressure in $\mathrm{mmHg}$ vs time in minute. $T_{0}$ is the starting point of the forecast window. The top plots are of patients who developed $A H E(H)$ after $T_{0}$. The bottom plots are of patients who did not have AHE (C).
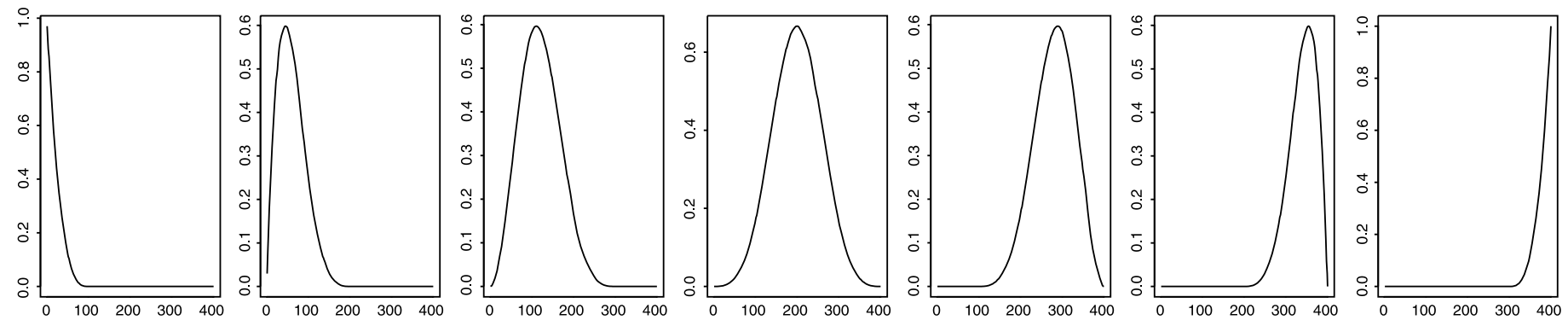

Figure 2. Plot of cubic B spline basis with three internal knots.

$\widehat{\alpha_{i k}}$ is the least square estimate by minimizing

$$
\sum_{j=1}^{n_{i}}\left(\sum_{k=1}^{K} \alpha_{i k} B_{k}\left(t_{j}\right)-A B P M_{i}\left(t_{j}\right)\right)^{2}
$$

To select the smoothing parameter, a generalized cross validation criterion $\widehat{G C V}_{i}(K)=\widehat{R S S}(i, K) /\left(n_{i}+1-\right.$ $2.5(K-1))^{2}$ is calculated for each $i, i=1, \ldots, N$, where

$$
\widehat{R S S}(i, K)=\sum_{j=1}^{n_{i}}\left(\sum_{k=1}^{K} \widehat{\alpha_{i k}} B_{k}\left(t_{j}\right)-A B P M_{i}\left(t_{j}\right)\right)^{2}
$$

and 2.5 is a penalty parameter (Stone et al., 1997). The smoothing parameter $K$ is selected by minimizing
$\sum_{i=1}^{N} \widehat{G C V}_{i}(K)$, where $N$ is 70 to predict Test Set A by adding Test Set A to the training set, and $N$ is 100 to predict Test Set B. Because of the different lengths of the training datasets, the smoothing parameter selection is done first on the minimal common interval of length of $T=656$. The remaining intervals are then assigned equally spaced knots generated from the common interval.

The Training Set, Test Set A and Test Set B are fitted with the selected $K$, and the result of matrices $\left(\alpha_{i k}\right)$ are denoted as $(\alpha)_{60, K}^{T},(\alpha)_{10, K}^{A}$ and $(\alpha)_{40, K}^{B}$, respectively.

These matrices are considered to be a discretization of the MAP curves and will preserve the features of these curves that can be used to carry out discrimination analysis. 


\section{RANK-BASED DISCRIMINATION}

A simple rank-based discrimination was developed from the Training Set and used to predict Test Set A. We first select those columns in $(\alpha)_{60, K}^{T}$ that have high discriminating power to distinguish AHE cases. We rank each column separately and then look at the distribution of AHE and non-AHE cases. Those columns with a high concentration of AHE or non-AHE cases at the top or bottom are considered to have high discriminatory power.

Let $I=\left(i_{1}, \ldots, i_{60}\right)$, where $i_{j}=-1$, for $j=1, \ldots, 30$ and 1 otherwise; $\overrightarrow{\alpha_{k}}=\left(\alpha_{1 k}, \ldots, \alpha_{60 k}\right)$ be the $k^{\text {th }}$ column of $(\alpha)_{60, K}^{T}$. Define $(1(k), \ldots, 60(k))$ as the order statistic generated from $\overrightarrow{\alpha_{k}}$, where $i(k)$ is the original location of $\alpha_{i(k) k}$ in $\overrightarrow{\alpha_{k}}$.

Then $I_{(k)}=\left(i_{1(k)}, \ldots, i_{60(k)}\right)$ is a transformation of $\overrightarrow{\alpha_{k}}$, where the entries of ordered $\overrightarrow{\alpha_{k}}$ become -1 for those corresponding to AHE cases and 1 for non-AHE cases. Large absolute values of $\sum_{j=1}^{30} j(k)$ and $\sum_{j=31}^{60} j(k)$ indicate a high concentration of similar groups at the top or bottom. A discriminant test statistic is defined as $\delta_{k}=\mid \sum_{j=1}^{30} j(k)-$ $\sum_{j=31}^{60} j(k) \mid$ for $\overrightarrow{\alpha_{k}}$. A large value of $\delta_{k}$ will indicate a high discrimination power of $\overrightarrow{\alpha_{k}}$.

\subsection{Application to Test Set A}

Here the rank base approach was applied to the Test Set A. To find which columns have the highest discrimi-

Table 1. $\delta_{k}$ from $\alpha_{60, K}^{T}$

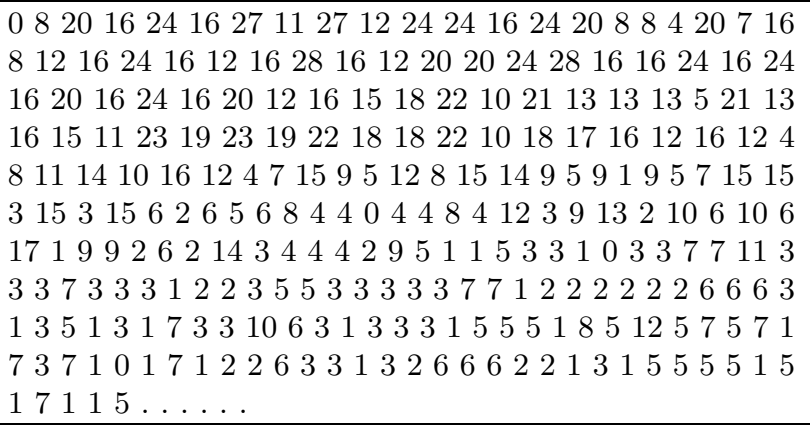

Table 2. Ordering the corresponding columns in Test Set $A$ matrix $(\alpha)_{10, K}^{A}$. The predicted " $H$ " is in bold

\begin{tabular}{|c|c|c|c|c|c|c|c|c|c|c|c|c|c|c|c|}
\hline $\mathrm{K}=$ & 5 & 7 & 9 & 11 & 12 & 14 & 25 & 29 & 34 & 35 & 38 & 40 & 44 & 63 & 65 \\
\hline & 110 & 110 & 110 & 110 & 102 & 110 & 101 & 101 & 101 & 101 & 101 & 101 & 101 & 102 & 104 \\
\hline & 109 & $\overline{101}$ & 101 & 102 & 109 & 101 & 109 & 110 & 109 & 110 & 102 & 109 & 109 & 104 & 101 \\
\hline & 104 & 104 & 109 & 101 & 110 & 109 & 110 & 109 & 110 & 109 & 109 & 110 & 104 & 101 & 110 \\
\hline & 102 & 102 & 102 & 104 & 101 & 104 & 102 & 108 & 104 & 102 & 104 & 104 & 102 & 110 & 102 \\
\hline & 108 & 108 & 108 & 108 & 107 & 108 & 108 & 104 & 108 & 108 & 108 & 108 & 108 & 105 & 105 \\
\hline & 103 & 103 & 103 & 103 & 103 & 103 & 103 & 103 & 103 & 105 & 105 & 103 & 103 & 103 & 103 \\
\hline & 106 & 106 & 106 & 106 & 106 & 106 & 106 & 106 & 106 & 106 & 106 & 106 & 106 & 106 & 106 \\
\hline
\end{tabular}

nation power from the training matrix $(\alpha)_{60, K}^{T}, \delta_{k}$ was calculated for each column. The result is in Table 1.

The largest $\delta_{k}$ 's are associated with columns $5,7,9,11$, $12,14,25,29,34,35,38,40,44,63,65$. The corresponding columns from matrix $(\alpha)_{10, K}^{A}$ were then selected and ordered separately. The results from application to Test Set A are displayed in Table 2. Except for column 29, every ordered column puts cases 101, 102, 104, 109 and 110 at the top portion and predicts them as AHE cases. In column 29, cases 101, 102, 108, 109, 110 are predicted as AHE cases. The entry for Test A received a full score on the first attempt.

\section{LOGISTIC REGRESSION AND CROSS-VALIDATION}

For Test Set B, the ranking approach did not generate a conclusive result. Therefore, a different approach was taken. By assigning 1 to AHE cases and 0 to non-AHE cases, the status could be naturally linked to $(\alpha)_{60, K}^{T}$ with logistic regression. The problem becomes how to search for columns from $(\alpha)_{60, K}^{T}$ that could be used to predict the outcome, a classical "best subsets" selection problem. There are many potential algorithms (Burnham and Anderson, 2002); here, we used a double cross-validation approach. Only about 650 minutes of data immediately preceding $T_{0}$ were used in this prediction since logistic regression has difficulty with missing data. A possible future extension would be to see whether imputing these missing data will improve the prediction.

\subsection{Leave a row out}

First, we will define criteria that could be used to select appropriate columns.

Denote $Y=\left(y_{1}, \ldots, y_{60}\right)^{t}$ and $(\alpha)_{60, K}^{T}=\left(\alpha_{1 .}, \ldots, \alpha_{60} .\right)^{t}$, where $y_{i}=1$ if $i$ th patient is AHE, or 0 otherwise and $\alpha_{i}$. is a vector of coefficients of $i^{\text {th }}$ MAP curve. For each $i$, remove $y_{i}$ from $Y, \alpha_{i}$. from $(\alpha)_{60, K}$. Fit the remainder $Y_{-i}$ to $(\alpha)_{-i}^{t}$ with logistic regression; the coefficient is denoted as $\beta_{-i}$. Then, $y_{i}$ can be predicted by $\hat{y}_{i}=\exp \left(\beta_{-i} \alpha_{i}\right.$. $) /(1+$ $\exp \left(\beta_{-i} \alpha_{i}\right)$.

Repeating this procedure for all $i, i=1, \ldots, 60$ in the Training Set, we obtain the prediction of $Y$ by $\hat{Y}=$ $\left(\hat{y}_{1}, \ldots, \hat{y}_{60}\right)$. 
Table 3. Prediction of status of Test Set $B$ and the true status. The true status was known after competition closed. Thirty three out of 40 were correctly predicted

\begin{tabular}{|r||r|r|r|r|r|r|r|r|r|r|r|r|r|r|r|r|r|r|r|r|}
\hline \hline Case & 201 & 202 & 203 & 204 & 205 & 206 & 207 & 208 & 209 & 210 & 211 & 212 & 213 & 214 & 215 & 216 & 217 & 218 & 219 & 220 \\
\hline \hline Prediction & $\mathrm{C}$ & $\mathrm{C}$ & $\mathrm{H}$ & $\mathrm{C}$ & $\mathrm{C}$ & $\mathrm{C}$ & $\mathrm{C}$ & $\mathrm{C}$ & $\mathrm{H}$ & $\mathrm{C}$ & $\mathrm{C}$ & $\mathrm{C}$ & $\mathrm{C}$ & $\mathrm{H}$ & $\mathrm{C}$ & $\mathrm{C}$ & $\mathrm{H}$ & $\mathrm{H}$ & $\mathrm{C}$ & $\mathrm{C}$ \\
\hline True & $\mathrm{C}$ & $\mathrm{H}$ & $\mathrm{H}$ & $\mathrm{C}$ & $\mathrm{C}$ & $\mathrm{C}$ & $\mathrm{H}$ & $\mathrm{C}$ & $\mathrm{H}$ & $\mathrm{C}$ & $\mathrm{C}$ & $\mathrm{C}$ & $\mathrm{C}$ & $\mathrm{H}$ & $\mathrm{C}$ & $\mathrm{C}$ & $\mathrm{H}$ & $\mathrm{H}$ & $\mathrm{C}$ & $\mathrm{C}$ \\
\hline \hline Case & 221 & 222 & 223 & 224 & 225 & 226 & 227 & 228 & 229 & 230 & 231 & 232 & 233 & 234 & 235 & 236 & 237 & 238 & 239 & 240 \\
\hline \hline Prediction & $\mathrm{C}$ & $\mathrm{C}$ & $\mathrm{H}$ & $\mathrm{C}$ & $\mathrm{H}$ & $\mathrm{H}$ & $\mathrm{H}$ & $\mathrm{C}$ & $\mathrm{C}$ & $\mathrm{C}$ & $\mathrm{C}$ & $\mathrm{C}$ & $\mathrm{C}$ & $\mathrm{H}$ & $\mathrm{C}$ & $\mathrm{C}$ & $\mathrm{C}$ & $\mathrm{H}$ & $\mathrm{C}$ & $\mathrm{C}$ \\
\hline True & $\mathrm{C}$ & $\mathrm{H}$ & $\mathrm{H}$ & $\mathrm{H}$ & $\mathrm{H}$ & $\mathrm{C}$ & $\mathrm{C}$ & $\mathrm{C}$ & $\mathrm{C}$ & $\mathrm{C}$ & $\mathrm{C}$ & $\mathrm{C}$ & $\mathrm{C}$ & $\mathrm{H}$ & $\mathrm{C}$ & $\mathrm{C}$ & $\mathrm{C}$ & $\mathrm{H}$ & $\mathrm{H}$ & $\mathrm{C}$ \\
\hline
\end{tabular}

We then define $C V F_{c}(\alpha)=$ mean $|Y-\hat{Y}|$ and $C V F_{d}(\alpha)=$ number of $\hat{y}_{i}$ such that $\left|y_{i}-\hat{y}_{i}\right|<0.5$. $C V F_{c}(\alpha)$ is a continuous measure for closeness of $\hat{Y}$ to $Y$, while the discrete measure of $C V F_{d}(\alpha)$ counts how many correct predictions are made if we assign 1 if $\hat{y}_{i}>0.5$ and 0 otherwise.

These two statistics will be used to guide the selection of columns from $(\alpha)_{60, K}^{T}$.

\subsection{Leave a column out}

Starting with full columns of $(\alpha)_{60, K}^{T}$, we delete less optimized columns repeatedly until no further improvement can be made.

Denote $(\alpha)_{60, K}^{T}=(\alpha \cdot 1, \ldots, \alpha \cdot K)$, where $\alpha_{\cdot k}$ is $k^{\text {th }}$ column. For each $k=1, \ldots, K$, remove $\alpha_{\cdot k}$ from $(\alpha)_{60, K}^{T}$ and the remainder is $(\alpha)_{-k} . C V F_{d}\left(\alpha_{-k}\right)$ and $C V F_{c}\left(\alpha_{-k}\right)$ are then calculated for the remainder matrix $(\alpha)_{-k}$. This procedure will generate vectors $\left(C V F_{d}\left(\alpha_{-1}\right), \ldots, C V F_{d}\left(\alpha_{-K}\right)\right)$ and $\left(C V F_{c}\left(\alpha_{-1}\right), \ldots, C V F_{c}\left(\alpha_{-K}\right)\right)$. From Section 4.1, the largest $C V F_{d}\left(\alpha_{-k}\right)$, or smallest $C V F_{c}\left(\alpha_{-k}\right)$ indicates that removing the corresponding column will produce a better prediction. We start with full columns and delete a column each time with the following strategies: 1) remove the column with the largest $\left.C V F_{d}\left(\alpha_{-k}\right) ; 2\right)$ if there are ties, remove the column with the smallest $C V F_{c}\left(\alpha_{-k}\right)$. This procedure removes one column, say $k_{1}$ with recorded $C V F_{d}\left(\alpha_{\cdot k_{1}}\right),\left(C V F_{c}\left(\alpha_{\cdot k_{1}}\right)\right)$.

The procedure is repeated for each remainder matrix until no further improvement can be made in $C V F_{d}\left(\alpha_{\cdot k_{1}}\right), C V F_{d}\left(\alpha_{\cdot k_{2}}\right), \ldots$ The remaining columns are considered to be the best prediction columns and the remainder matrix is denoted as $(\alpha)_{60, K-l}$ where $l$ columns are removed. A logistic regression of $Y$ on $(\alpha)_{60, K-l}$ is carried out to get coefficient $\beta_{K-l}$. Let $(\alpha)_{40, K-l}^{B}$ be the reminder matrix that resulted from $(\alpha)_{40, K}^{B}$, where the same $l$ columns are removed. $Y^{B}$ is then predicted by

$$
Y^{B}=e^{(\alpha)_{40, K-l}^{B} \beta_{K-l}} /\left(1+e^{(\alpha)_{40, K-l}^{B} \beta_{K-l}}\right)
$$

\subsection{Application to Test Set B}

One curve was removed from the training set because of a large missing segment near the beginning time point. Thus, 59 curves were used in the cross-validation process. The initial leave-a-row-out procedure returns the largest
$C V F_{d}\left(\alpha_{40}\right)=48$. Column 40 was first removed. Sequentially, columns 26,33 , and 44 were removed. The procedure returns $C V F_{d}\left(\alpha_{22}\right)=58$. After removing column 22 , the next run did not provide further improvement.

Denote $l=(22,26,33,44)$. The prediction $Y^{B}$ was then calculated with formula 1 in Section 4.2.

Simply predicting AHE cases by $\left|y_{i}^{B}-1\right|<0.5$ overestimated the number of AHE cases, the predicted AHE cases were over the limit specified in the competition instruction. For all $y_{i}^{B} \in[0.485,0.515]$, we used the rank analysis, as discussed in Section 3, to window the number of predicted AHE cases. The prediction of status for each case in Test Set $\mathrm{B}$ is presented in Table 3 . The procedure correctly predicts 33 out of 40 and our entry scored eighth in the competition.

\section{FURTHER EXPLORATION POST-COMPETITION}

In previous sections, predictions for Test Sets A and B were made using different methods. After the competition, the true status was available for both test sets. Nevertheless, we attempted to find a method able to predict both sets well.

The logistic regression approach in Section 4 used only common-length data proximal to $T_{0}$ because of missing data. The length of the data segment was about 656 minutes. Thus, a large amount of information was available but not used in the analysis. To use more information and with the insight that the magnitude of the MAP likely plays an important role in the prediction of Test Set A, we added $5 \%$, $15 \%, 25 \%, 50 \%$, and $75 \%$ quantiles of each curve to the discretization matrices $(\alpha)_{60, K}^{T},(\alpha)_{10, K}^{A}$ and $(\alpha)_{40, K}^{B}$, respectively. To each matrix was added an additional five columns, making the total number 52 .

It is a well known fact that in model selection, the best set might be biased toward the training set (Burnham and Anderson, 2002). Therefore, we selected the second or third best subsets in the cross validation process.

In the cross validation to determine the best fit, we used a "one out N" approach for predicting $Y$. In the literature, various " $\mathrm{m}$ out $\mathrm{N}$ " approaches are discussed as a way to improve the accuracy of prediction (Breiman and Spector, 1992). We thought this might be suitable here, since the final prediction was carried out for a group of patients. However, the requirement for a non-singular matrix in the logistic 
Table 4. Prediction of status of Test Set B post competition. Thirty five out of 40 were correctly predicted

\begin{tabular}{|r||r|r|r|r|r|r|r|r|r|r|r|r|r|r|r|r|r|r|r|r|}
\hline \hline Case & 201 & 202 & 203 & 204 & 205 & 206 & 207 & 208 & 209 & 210 & 211 & 212 & 213 & 214 & 215 & 216 & 217 & 218 & 219 & 220 \\
\hline \hline Prediction & $\mathrm{C}$ & $\mathrm{H}$ & $\mathrm{H}$ & $\mathrm{C}$ & $\mathrm{C}$ & $\mathrm{H}$ & $\mathrm{H}$ & $\mathrm{C}$ & $\mathrm{H}$ & $\mathrm{C}$ & $\mathrm{C}$ & $\mathrm{C}$ & $\mathrm{C}$ & $\mathrm{H}$ & $\mathrm{C}$ & $\mathrm{C}$ & $\mathrm{H}$ & $\mathrm{H}$ & $\mathrm{C}$ & $\mathrm{C}$ \\
\hline True & $\mathrm{C}$ & $\mathrm{H}$ & $\mathrm{H}$ & $\mathrm{C}$ & $\mathrm{C}$ & $\mathrm{C}$ & $\mathrm{H}$ & $\mathrm{C}$ & $\mathrm{H}$ & $\mathrm{C}$ & $\mathrm{C}$ & $\mathrm{C}$ & $\mathrm{C}$ & $\mathrm{H}$ & $\mathrm{C}$ & $\mathrm{C}$ & $\mathrm{H}$ & $\mathrm{H}$ & $\mathrm{C}$ & $\mathrm{C}$ \\
\hline \hline Case & 221 & 222 & 223 & 224 & 225 & 226 & 227 & 228 & 229 & 230 & 231 & 232 & 233 & 234 & 235 & 236 & 237 & 238 & 239 & 240 \\
\hline \hline Prediction & $\mathrm{C}$ & $\mathrm{H}$ & $\mathrm{H}$ & $\mathrm{H}$ & $\mathrm{C}$ & $\mathrm{H}$ & $\mathrm{C}$ & $\mathrm{C}$ & $\mathrm{C}$ & $\mathrm{H}$ & $\mathrm{C}$ & $\mathrm{C}$ & $\mathrm{C}$ & $\mathrm{H}$ & $\mathrm{H}$ & $\mathrm{C}$ & $\mathrm{C}$ & $\mathrm{H}$ & $\mathrm{H}$ & $\mathrm{C}$ \\
\hline True & $\mathrm{C}$ & $\mathrm{H}$ & $\mathrm{H}$ & $\mathrm{H}$ & $\mathrm{H}$ & $\mathrm{C}$ & $\mathrm{C}$ & $\mathrm{C}$ & $\mathrm{C}$ & $\mathrm{C}$ & $\mathrm{C}$ & $\mathrm{C}$ & $\mathrm{C}$ & $\mathrm{H}$ & $\mathrm{C}$ & $\mathrm{C}$ & $\mathrm{C}$ & $\mathrm{H}$ & $\mathrm{H}$ & $\mathrm{C}$ \\
\hline
\end{tabular}

regression limits $\mathrm{m}$ to not more than 6 . We attempted $m=3$ here. To try out all possible combinations of 3 out of 52 columns would be infeasible, so the following sets were used.

Let, $s_{i}=(i, 20+i, 40+i), i=1, \ldots, 20$. Similar to Section 4.1 , For each $i$, remove $y_{s_{i}}$ from $Y, \alpha_{s_{i}}$ from $(\alpha)_{60, K}$. Fit the remainder $Y_{-s_{i}}$ to $(\alpha)_{-s_{i}}^{t}$ with logistic regression; the coefficient is denoted as $\beta_{-s_{i}}$. Then, $y_{s_{i}}$ can be predicted by $\hat{y}_{s_{i}}=\exp \left(\beta_{-s_{i}} \alpha_{s_{i}}\right) /\left(1+\exp \left(\beta_{-s_{i}} \alpha_{s_{i}}\right) . C V F_{c}(\alpha)=\right.$ mean $|Y-\hat{Y}|$ and $C V F_{d}(\alpha)$ are defined as same as in Section 4.1 by just replacing $i$ with $s_{i}$.

Then we followed the same procedure in Section 4.2, but each time we removed the column with the secondbest fit or third-best fit instead of the best fit. Here the best fit used the smallest $C V F_{c}(\alpha)$, while the second- or third-best fit used the second or third smallest $C V F_{c}(\alpha)$. We found that removing the column with the third-best fit yielded the best fit with the column subset of $(2,3,10,14$, $16,19,24,26,27,35,36,37,38,39,42,43,44,45,47)$ and columns corresponding to the $5 \%, 15 \%$, and $25 \%$ quantiles. With this subset, we can predict correctly all outcomes in Test Set A and 35 out of 40 in Test Set B. The result is shown in Table 4.

\section{CONCLUSION REMARKS}

This manuscript proposes a two-stage method for predicting AHE from continuous MAP data. In statistical literature, most two-stage approaches can eventually be improved by joining two steps into a simultaneous process. The twostage approach proposed here is different from these types of approaches. B-Spline fitting is used to find the best fit for the curve, and the second stage finds the best predictors from the fitted coefficients. The best predictors may not necessarily be the best base to fit the curve.

The proposed approach has the potential to be employed in searching for hidden clinical features that may not be easily seen from a digitized wave dataset. Indeed, our approach does not assume any prior knowledge about the dataset or its clinical interpretation.

It may be possible to do much better in predicting clinical events if we utilize other available clinical telemetry dataheart rate, systolic and diastolic pressure - but we have not explored how much these add, or even if MAP was the optimal single channel.
In addition, the proposed methods could be further improved by careful consideration of how to assess variances in the datasets and estimates.

The training datasets have various lengths, from 656 to 14,986 minutes. The logistic regression and cross-validation methods we used require each dataset to be the same length. Therefore, only limited data segments with a common minimum length were used in the logistic regression and crossvalidation. This limitation can probably be overcome in future work.

\section{Received 20 December 2010}

\section{REFERENCES}

Moody, G. B. and Lehman, L. H. (2009). Predicting acute hypotensive episodes: The 10th annual PhysioNet/Computers in Cardiology Challenge. Computers in Cardiology 36.

DE Boor, C. (2001). A Practical Guide to Splines. Springer, New York. MR1900298

JIN, K. (1992). Empirical smoothing parameter selection in adaptive regression. Ann. Statist. 20 1844-1874. MR1193315

Stone, C. J., Hansen, M., Kooperberg, C., and Truong, Y. K. (1997). Polynomial splines and their tensor products in extended linear modeling. Ann. Statist. 25 1371-1425. MR1463561

Burnham, K. P. and Anderson, D. R. (2002). Model Selection and Multimodel Inference: A Practical Information-Theoretic Approach, 2nd ed. Springer, New York. MR1919620

Breiman, L. and Spector, P. (1992). Submodel selection and evaluation in regression: The X-random case. International Statistical Review 60 291-319.

Kun Jin

Division of Biometrics I

FDA/CDER, WO21, Rm 4622

10903 New Hampshire Ave

Silver Spring, MD 20993

USA

E-mail address: kun.jin@fda.hhs.gov

Norman Stockbridge

Division of Cardiovascular and Renal Products

FDA/CDER

10903 New Hampshire Ave

Silver Spring, MD 20993

USA

E-mail address: norman.stockbridge@fda.hhs.gov 